\title{
Lösungsmöglichkeiten nach fehlgeschlagenen Osteosynthesen: Hüftkopf und Schenkelhals
}

\author{
Felix Bonnaire, Thomas Lein, Henning Röhl
}

\section{Zusammenfassung}

Partielle oder vollständige Hüftkopfnekrosen, verbleibende Hüftkopfdefekte und Gelenkinkongruenzen und -instabilitäten, intraartikuläre freie Fragmente, Bewegungseinschränkungen und posttraumatische Arthrosen sind nach Osteosynthesen bei Hüftkopffrakturen die häufigsten Problemstellungen. Da zumeist jüngere Patienten von den Hüftkopf(luxations)frakturen betroffen werden, sind gelenkoder zumindest knochenerhaltende Lösungen grundsätzlich zu favorisieren gegenüber primär resezierenden Verfahren und Endoprothesen. Die Indikationen sind ausschließlich individuell $\mathrm{zu}$ stellen und beinhalten von der arthroskopischen Exploration bis zur Implantation einer zementfreien oder zementierten Totalendoprothese eine breite Eingriffspalette. Vor allem schenkelkopf- und halserhaltende Prothesen bieten bei sehr jungen Patienten annehmbare Alternativen zum totalen resezierenden Gelenkersatz. Nach operativer Behandlung und Fehlschlägen nach Schenkelhalsfrakturen des älteren Menschen kommen nur in Ausnahmefällen Reosteosynthesen in Betracht. Für diese Patienten ist eine prothetische Versorgung mit Duokopf- oder Totalendoprothese grundsätzlich die sichere Variante. Bei jüngeren Patienten sind Rettungsoperationen mit Umstellungsoperationen zum Gelenkerhalt vorzuziehen. In aussichtslosen Fällen sollte man sich bei diesen Patienten zu einem funktionell noch günstigen Zeitpunkt zu einem möglichst knochenerhaltenden Gelenkersatz entscheiden.

\section{Einleitung}

\section{Hüftkopffrakturen}

Hüftkopffrakturen gehen häufig mit Luxationen im Hüftgelenk einher. Sie sind seit der Einführung der Sicherheitsgurte in PKW insgesamt zurückgegangen, ereignen sich in der Regel nach Hochrasanztraumen und gehen mit mehr oder weniger manifesten Knorpelschäden einher. Der Mechanismus der Entstehung ist eine axiale Stauchung des Oberschenkels mit mehr oder weniger Flexion in der Hüfte verbunden mit einer Ad- oder Abduktion des Oberschenkels. Es resultieren Druck- und Scherkräfte auf den Schenkelkopf, bei Adduktion eher am un-

OP-JOURNAL 2004; 20: 134-140

(c) Georg Thieme Verlag KG Stuttgart · New York teren Kopfquadranten, bei Abduktion eher am Schenkelhals. Die Luxationsfrakturen werden in der Regel nach Pipkin klassifiziert. Da Impressionensfrakturen am Femurkopf als Begleitverletzungen eine zusätzliche, die Prognose wesentlich verschlechternde Auswirkung haben, hat die AO diese Frakturen zusätzlich in ihre Klassifikation mit aufgenommen.

Impressionen am Femurkopf werden allenfalls im CT an einer Entrundung der Kalotte oder intraoperativ erkannt, Einblutungen und Mikrofrakturen nur im MRT, sodass die Gesamtschädigung des Hüftkopfes und der azetabulären Gelenkseite meist im Ungewissen bleibt.

Bei Luxationen reißt das Ligamentum capitis femoris mit dem Ramus acetabularis, der Zentralarterie, die aus der Arteria obturatoria interna gespeist wird, immer ab. Diese Tatsache hat für die Durchblutung des Femurkopfes nur eine unterge-

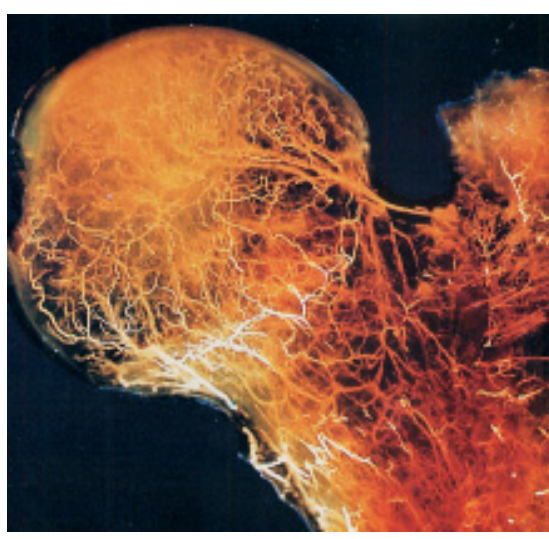

Abb.1 Durchblutungsverhältnisse eines 27jährigen, jungen Mannes: Die Femurkopfdurchblutung kommt von den lateralen Epiphysengefäßen; über ein ausgedehntes Anastomosennetz ist eine dreidimensionale Versorgung möglich. Direkt unter der Kopfkalotte sind die Arterien Endarterien, die durch Kontusion oder Fraktur verletzt und obturiert werden können. Aus Henry V. Crock: An Atlas of vascular Anatomy of the skeleton and spinal cord [4].

ordnete Bedeutung, da von dieser Arterie nur allenfalls ein Fünftel der Durchblutung des Hüftkopfes und nur in den zentralen, nicht belasteten Anteilen des Hüftopfes ausgeht [10]. Entscheidend kann sich der Verlust der Arterie bei einer Reperfusion des Hüftkopfes auswirken, wenn gleichzeitig eine Schenkelhalsfraktur vorliegt oder aber eine kontusionsbedingte Perfusionsstörung. Hüftkopfimpressionen oder -frakturen ohne Luxation können ebenfalls mit Perfusionsstörungen einhergehen. Die Durchblutung des Femurkopfes erfolgt über ein dreidimensionales Netzwerk im Wesentlichen durch die von dorsal eintretenden 3-5 lateralen Epiphysengefäße, die bei Frakturen und Impressionen direkt geschädigt werden können (Abb.1). Da sie subchondral als Endgefäße fungieren, ist diese Region am stärksten gefährdet. Eine Revaskularisation kann über die Umgebung und vor allem von metaphysär erfolgen. 


\section{Behandlungsgrundsätze}

Erstes Ziel in der Behandlung ist die Reposition der Luxation und der Fraktur. Danach ist ein CT hilfreich zur weiteren Behandlungsplanung. Beurteilt werden die Kongruenz des Hüftkopfes im Gelenk, eine Impression des Azetabulums, die Stellung der Fraktur am Hüftkopf, Impressionen am Hüftkopf und Hinweise auf freie Gelenkkörper oder KnorpelKnochenfragmente.

Letztere können arthroskopisch näher beurteilt oder gar entfernt werden oder werden anlässlich einer chirurgischen Gelenkluxation in Verbindung mit einer Osteosynthese oder Anhebung einer Impression mitbehandelt. Die Refixation von Gelenkanteilen erfolgt in der Regel unter Luxationsbedingungen direkt mit Minifragmentschrauben, die unter Knorpelniveau versenkt werden oder mit resorbierbaren Schrauben und Stiften. Auch indirekte Verschraubungen mit Zugschrauben sind möglich. Gelingt keine stufenlose, stabile Refixation des Fragmentes, ist eine Entfernung auch bei größeren (Pipkin II Frakturen) Defekten günstiger für den Verlauf als eine schlechte Refixation.
Im Verlauf der Behandlung sind Komplikationen häufig: partielle oder totale Hüftkopfnekrosen, Schraubenlockerung, Schmerzen und Bewegungseinschränkung sind die häufigsten und die posttraumatische Arthrose des Hüftgelenkes steht am Ende der Entwicklung. Nicht selten tritt auch eine Schenkelhalsnekrose ein. Auf diese möglichen Komplikationen sollte der Patient am Beginn der Behandlung hingewiesen werden, um seine Kooperation in der Therapie zu optimieren.

Zu Beginn eines komplizierten Verlaufs sollte frühzeitig eine möglichst vollständige bildgebende Diagnostik einschließlich CT- und/oder eine MRT-Untersuchung die Verhältnisse an den Gelenkflächen und die Perfusionsverhältnisse klären. Reperfusionen sind bis zu 3 Monaten möglich und sollten so lange beobachtet werden. Bei anhaltender Perfusionsminderung und drohendem Verlust der mechanischen Kompetenz des betroffenen Areals sollte man revaskularisierende Maßnahmen überdenken. Inkongruenzen der Gelenkoberfläche müssen wenn möglich sofort behandelt werden, da ohne Korrektur die Arthrose rapide fortschreitet.
Die mit Abstand häufigste Lösung nach fehlgeschlagenen Osteosynthesen bei Hüftkopffrakturen ist die Hüftkopfentfernung und Implantation einer zementfreien Hüftprothese. Vor allem aber, wenn ganz junge Patienten betroffen sind, sollte man alle Möglichkeiten der gelenkerhaltenden Maßnahmen in Erwägung ziehen und mit dem Patienten besprechen. Anhand von einigen Beispielen sollen die Behandlungsmöglichkeiten bei Fehlschlägen in der Frakturbehandlung erläutert werden:

Ein 38-jähriger kräftiger, übergewichtiger Patient zieht sich bei einem PKW-Unfall eine Pipkin-II-Fraktur zu. Die Reposition erfolgt sofort, im CT nach der Reposition ist eine Inkongruenz erkennbar, es erfolgt die offene Repostion und Fragmentverschraung mit 3,5 mm Schrauben. Postoperativ zunächst adäquater Verlauf, nach 3 Monaten weiter bestehende Schmerzen. Weiteres Abwarten einer Physiotherapiephase über 3 Monate, leichte, aber nicht entscheidende Besserung. Zunehmende Bewegungseinschränkung des Hüftgelenkes mit Beugekontraktur. Zunehmende Arthrosezeichen, Entscheidung zur Implantation

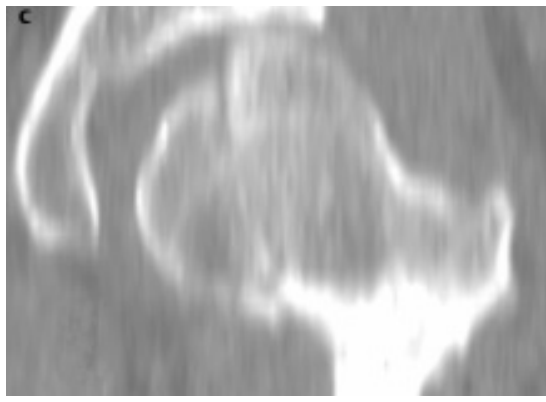

(c) Stufenbildung in der Frontalebene. Letzendlich verbleibt noch ein geringer Oberfächenanteil des Femurkopfes in der Hauptbelastungszone stehen. Daraufhin Indikation zur operativen Behandlung.
Abb. 2a-c CT-Untersuchung des 38-jährigen Patienten nach PKWUnfall nach der Reposition in der Notaufnahme: (a) Transversalschnitt zur Planung der sagittalen Schnitte. (b) Ein sagittaler Schnitt, der die Defektbildung bei der verbleibenden Dislokation ventral dokumentiert.
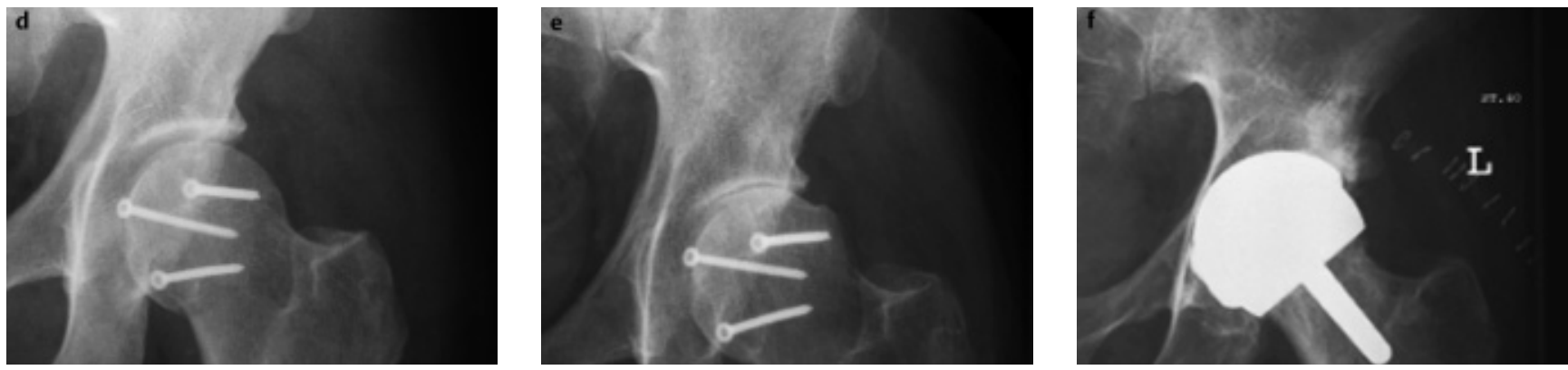

Abb. 2d-f Verlauf der Behandlung: (d) postoperative a.-p. Röntgenaufnahme: korrekte Reposition des Kalottenfragmentes nach direkter Verschraubung. Stufenlose Reposition in beiden Ebenen. (e) Röntgenbild nach 1 Jahr: beginnende Abstützungsreaktion am Schenkelhals und
Ausbildung einer zunehmenden Koxarthrose. (f) Bild nach 18 Monaten: zunehmende Beschwerden und Bewegungseinschränkung. Indikation zur kopf-und halserhaltenden Oberflächenprothese. 
einer Mc Minn-Prothese, die Oberschenkelkopf und -hals erhält, nach 18 Monaten. Besserung der Belastbarkeit aber keine Beschwerdefreiheit (Abb.2a-f).

48-jähriger Patient, Sturz als Dachdecker aus $6 \mathrm{~m}$ Höhe auf harten Untergrund, Pipkin-IV-Verletzung. Sofortige Reposition und CT-Kontrolle: Azetabulumfraktur und großes Hüftkopffragment, disloziert. Plattenosteosynthese des hinteren Randes mit Spongiosaplastik und Refixation des Kopffragmentes mit 2,7 mm Schrauben. Keine Schmerzfreiheit. Nach 3 Monaten vollständige Femurkopfnekrose und Implantation einer TEP (Abb.3a-f).

Grundsätzlich stehen in ähnlich gelagerten Fällen nach Hüftkopffrakturen bei weiterbestehender, nicht mehr adäquater Schmerzsymptomatik und Bewegungseinschränkung folgende diagnostischtherapeutischen Optionen zur Verfügung:
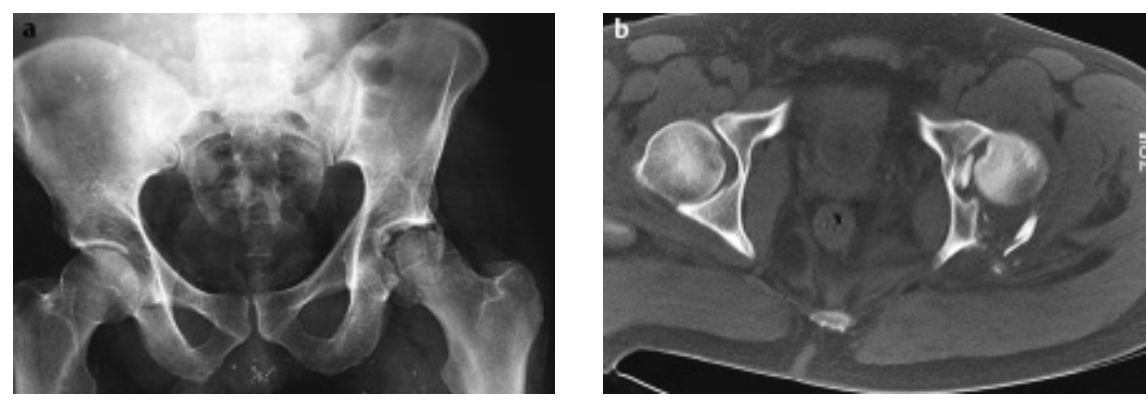

Abb.3au.b 48-jähriger Dachdecker mit Sturz aus $6 \mathrm{~m}$ Höhe auf harten Untergrund. Pipkin-IVVerletzung mit Femurkopffraktur und Zerstörung der dorsalen Pfannenwand. (a) Beckenübersichtsbild vom Unfalltag mit Luxation der linken Hüfte. (b) CT nach Reposition der Hüfte: schalenförmiges Kalottenfragment nachweisbar. Defekt in der dorsalen Pfannenwand. Indikation zur Verschraubung des Kalottenfragmentes und dorsalen Pfannenwandplastik mit Plattenosteosynthese.
Osteochondrale Sequestrierung: Anbohrung, Spongiosaunterfütterung und Refixation des Fragmentes.

Entrundung des Femurkopfes durch Einbruch in der Belastungsfläche: Osteochondrale autologe Transplantation (OATS), Umstellungsosteotomie zum Herausrotieren der zerstörten Gelenkfläche aus der Belastung, Oberflächenprothese (McMinn) [5].

Fortgeschrittene Hüftkopfnekrose: Druckscheibenprothese [7], zementfreie Totalendoprothese mit Hydroxylbeschichtung.

Posttraumatische Koxarthrose: Zementfreie Totalendoprothese mit Hydoxylapatitbeschichtung [6], Oberflächenprothese oder Druckscheibenprothese [5].

Die Ergebnisse mit den allein oberflächenersetzenden Prothesen mit MetallMetallpaarungen sind nach schlechten Erfahrungen mit früheren Typen (Wagner-Cup) heute auch langfristig ermutigend, sodass die große Zurückhaltung aus früheren Zeiten wegen hoher Lockerungsraten bei jungen Patienten nicht mehr gerechtfertigt ist [5-7].

\section{Schenkelhalsfrakturen}

Die Frakturen des jüngeren Menschen in der Zone des intrakapsulären Anteiles des Schenkelhalses entstehen ebenfalls nach Rasanztraumen mit hoher lokaler Energie und können auf die gleichen Mechanismen wie die Hüftkopffrakturen bei stärkerer Extension des Oberschenkels im Hüftgelenk auf Schermechanismen
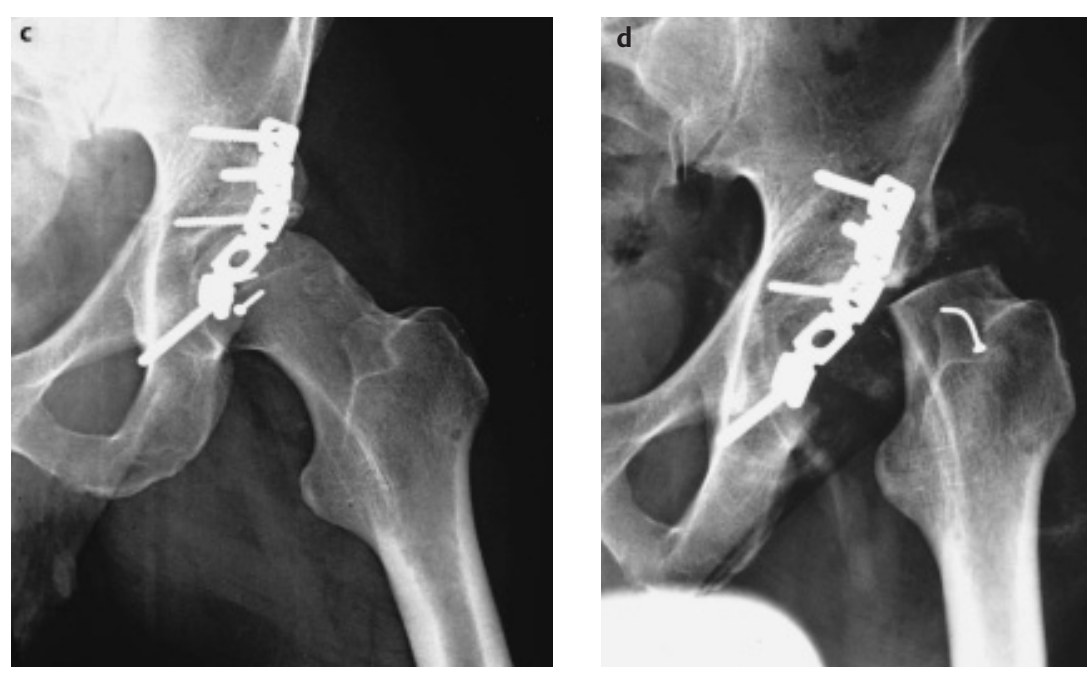

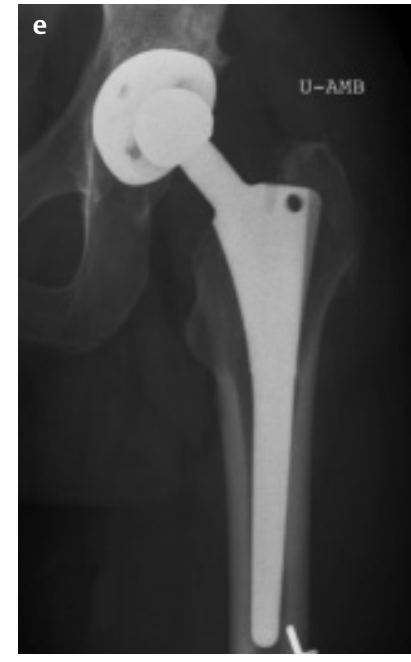

rand und der -wand. (d) Röntgenbild 3 Monate nach der Verletzung und Behandlung: vollständige Femurkopfnekrose. (e) Implantation einer zementfreien TEP nach Metallentfernung der Platte. Z. n. 3 Jahren.
Abb.3c-e Verlauf der Behandlung: (c) Röntgenbild nach Verschraubung des ventralen Kalottenfragmentes mit zwei 2,7 mm Schrauben mit guter Kongruenz im Gelenk. Spongiosaplastik am dorsalen Pfannen- 
zurückgeführt werden. Damit sind auch Kontusionen des Hüftkopfes mit Impressionen grundsätzlich möglich. Durch den Eintritt der lateralen Epiphysenarterien von dorsal und kranial unterhalb der Knorpelgrenze in den knorpeltragenden Femurkopf sind bei stärkeren Dislokationen Perfusionsstörungen des gesamten Femurkopfes oder des lateralen Anteils häufig und Femurkopfnekroseraten bis zu 50\% werden in Abhängigkeit vom Therapieverfahren und -zeitpunkt beschrieben [2].

Eine Perfusionsstörung ist jedoch nicht gleichbedeutend mit einer manifesten Femurkopfnekrose, da Reperfusionen in einem hohen Prozentsatz erfolgen können. Im jugendlichen Alter ist deshalb ein Erhaltungsversuch des Femurkopfes nahezu immer gerechtfertigt. Im Verlauf ist aber kontinuierlich auf Beschwerden und Bewegungseinschränkungen zu achten und bei entsprechendem Verdacht eine bildgebende Diagnostik (CT und MRT) zu veranlassen, damit die unter Hüftkopffrakturen genannten Möglichkeiten erörtert und frühzeitig, vor dem Eintritt einer schweren Funktionsstörung oder posttraumatischen Koxarthrose, vorgenommen werden können.

Beim älteren Patienten ist der Entstehungsmechanismus der Frakturen durch einen Sturz auf den Trochanter major mit einer axialen Stauchung des Schenkelhalses und je nach Sturz mehr auf die seitlichen oder dorsalen Anteile des Trochanters mit einer Außenrotation des Oberschenkels verbunden. Beim Überschreiten einer bestimmten Dislokationskraft von dorsal entsteht dorsal am Schenkelhals eine Impressionszone und ventral klaffen die Bruchanteile auseinander. Es resultiert eine zunehmende Instabilität, wobei beim Garden-III-Typ die dorsalen Frakturanteile noch in Kontakt bleiben, bei Fortschreiten der Dislokation (Garden IV) aber vollständig getrennt werden und in allen Positionen ohne Kontakt bleiben. Impressionen des Femurkopfes sind weniger zu erwarten, allerdings mit zunehmender Dislokation auch Perfusionsstörungen.

Als weitere Hauptkomplikation kann eine Pseudarthrose (10-25\%) des Schenkelhalses eintreten.

Die impaktierten und nicht dislozierten Frakturen werden im Allgemeinen operativ mittels Osteosynthese versorgt, die dislozierten Frakturen wegen zu erwartender Komplikationen meistens endoprothetisch. Im fortgeschrittenen Alter, bei entsprechend reduziertem Belastungswunsch, Osteoporose und anderen limitierenden Begleiterkrankungen entscheidet man sich bei eintretenden Femurkopfnekrosen, Pseudarthrosen oder Heilungen in Fehlstellung oder Verkürzung des Schenkelhalses leichter zum endoprothetischen Ersatz des Femurkopfes (Duokopfprothese) oder des gesamten Gelenkes (Totalendoprothese) in Form einer sofort voll belastbaren Prothese (Hybrid oder zementiert).

Typische Fehlschläge bei jüngeren Patienten mit ausgeprägtem Belastungswunsch sind in der Reihenfolge der Häufigkeit

1. die partielle oder komplette Femurkopfnekrose

2. die Schenkelhalspseudarthrose

3. die Verkürzung des Schenkelhalses

4. die Valgisierung des Schenkelhalses

5. die Außenrotationsstellung des Femur

\section{Lösungen}

Für die Perfusionsstörung des Schenkelkopfes und deren Behandlung gelten nahezu identische Grundsätze wie für die Femurkopffrakturen. Sämtliche dort aufgeführten Verfahren sind bei Partialnekrosen möglich. Die komplette Femurkopfnekrose hinterlässt aber einen grö-
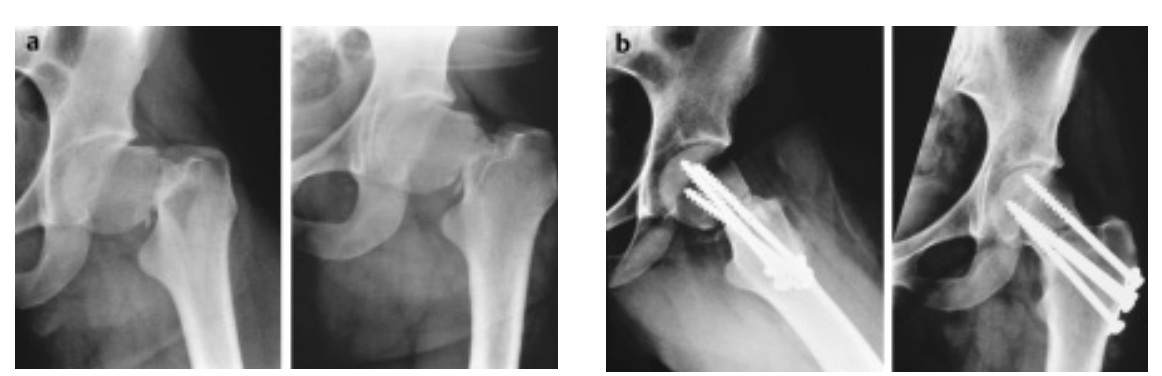

Abb.4a-b 28-Jährige Patienten, nach Trampen und Überfall aus fahrendem PKW geworfen. (a) Instabile Schenkelhalsfraktur Typ Garden IV. (b) Sofortversorgung nach Rettung etwa $10 \mathrm{~h}$ nach Ereignis mit Schraubenosteosynthese.
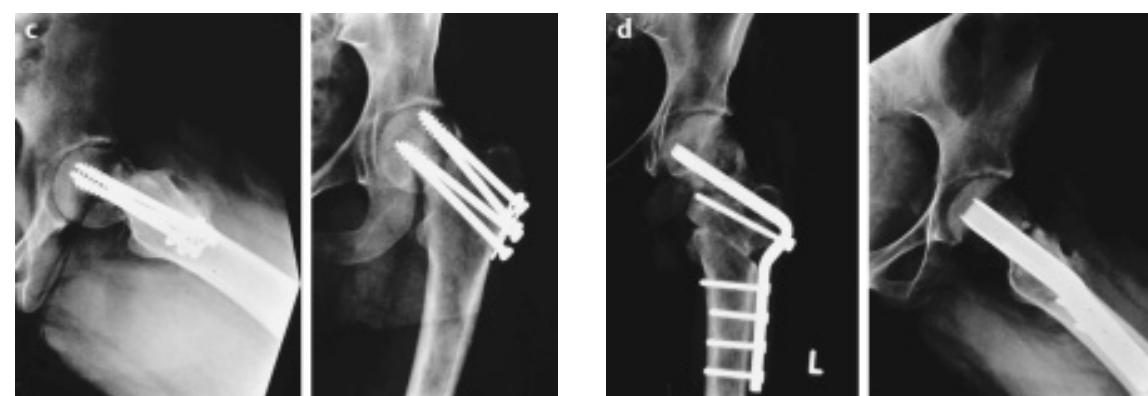

Abb.4c-d (c) Entwicklung einer Schenkelhalspseudarthrose nach 6 Monaten. Varusdislokation des Kopffragmentes (d) Intertrochantere, valgisierende Osteotomie, die letztendlich zur Ausheilung der Pseudarthrose führte.

ßeren Substanzdefekt am Femurkopf und häufig auch am Schenkelhals, sodass ein alleiniger Oberflächenersatz kann einen größeren Knochenverlust vermeiden helfen, die Langzeiterfahrungen

Vor dem Femurkopfkollaps sind aber auch invasivere Techniken wie z.B. gefäßgestielte Spantransplantationen vom Beckenkamm überlegenswert.

Schenkelhalspseudarthrosen werden mit einer intertrochanteren, valgisierenden Umstellungsosteotomie mit gutem Behandlungserfolg zur Heilung gebracht. eine Subluxation des Hüftkopfes un eine merkbare Inkongruenz im Gelenk auftritt, sollte bei einer stärkeren Valgisierung eine Revarisierung erfolgen. Außerdem werden bei zunehmender Valgidie Außenrotatoren der Hüfte nisch überlastet und es kann eine Femurkopfnekrose oder lokale Arthrose in der Hauptbelastungszone eintreten [7].

Das gleiche Problem stellt sich ein bei in stärkerer Valgusstellung osteosynthetisch versorgten Schenkelhalsfrakturen. 
$\mathrm{Zu}$ der Valgusstellung tritt häufig noch eine Außenrotation des Oberschenkels, wodurch Ursprung und Ansatz der pelvi-trochanteren Muskulatur soweit genähert werden, dass eine kräftige Kontraktion der Muskeln nicht mehr möglich ist. Es resultiert ein Watschelgang mit positivem Trendelenburg-Zeichen. Wenn noch eine relevante Schenkelhalsverkürzung eintritt, verschlechtert sich die Situation weiterhin.

Ziel der Korrektur sollte damit eine möglichst anatomische Stellung der Fraktur sein, wobei die Verkürzung des Schenkelhalses am schwierigsten und letzendlich nur mit einer Valgsierung effektiv auszugleichen ist. Bei jungen aktiven Patienten ist der Versuch einer Korrektur immer gerechtfertigt. Die Ergebnisse nach Umstellungsosteotomien und anschließen-
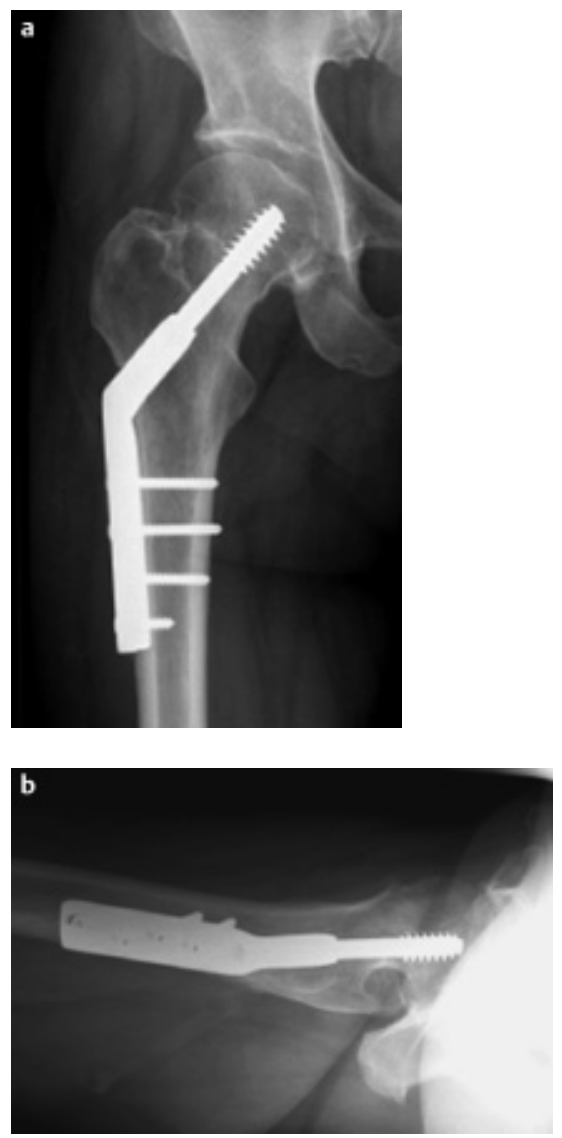

Abb.5a-b Röntgenbilder einer 40-jährigen Patientin mit valgisch eingestauchter Schenkelhalsfraktur rechts nach Fahrradsturz. Sofortversorgung mit DHS unter Belassen der Impaktion. Im axialen Bild(b) Retroversion des Femurkopfes erkennbar. Fraktur heilt problemlos aus, Patientin hat aber kontinuierlich Schmerzen und kann nicht auftreten. Schwerste Muskelinsuffizienz und Entwicklung einer Beugekontraktur. Vorstellung zum weiteren Prozedere. dem endoprothetischen Ersatz sind gleichwertig der primär endoprothetischen Lösung [9].

\section{Beispiele}

28-jährige Patientin, bei einem Überfall aus einem fahrenden PKW geworfen: Schenkelhalsfraktur Typ Garden IV. Sofortige Reposition und Schraubenosteosynthese. Es entwickelte sich eine Pseudarthrose: Durch eine valgisierende intertrochantere Osteotomie kam die Pseudarthrose zur Ausheilung (Abb.4a-d).

36-jähriger Patient, stürzt alkoholisiert in der Wohnung. Vorstellung $36 \mathrm{~h}$ nach Ereignis: Schenkelhalsfraktur Typ Garden IV: sofortige Osteosynthese. Im Verlauf Patient schlecht kooperierend, kontinuierlich Schmerzen. Nach 6 Monaten Diagnostik mit Knochensequenzszintigraphie, CT und MRT: Schenkelhalsnekrose mit Verkürzung und Pseudarthrose. Reosteosynthese mit intertrochanterer, valgisierender Osteotomie, Verlängerung des Schenkelhalses mit einem trikortikalen Span aus dem Beckenkamm. Postoperativ sofortige Symptomverbesserung. Ausheilung nach 6 Monaten in leichter Verkürzung (keine Bildserie vorhanden).

40-jährige Patientin mit Sturz vom Fahrrad, valgisch eingestauchte Fraktur vom Typ Garden I, Osteosynthese ohne Reposition der Valgusstellung und Außenrota-
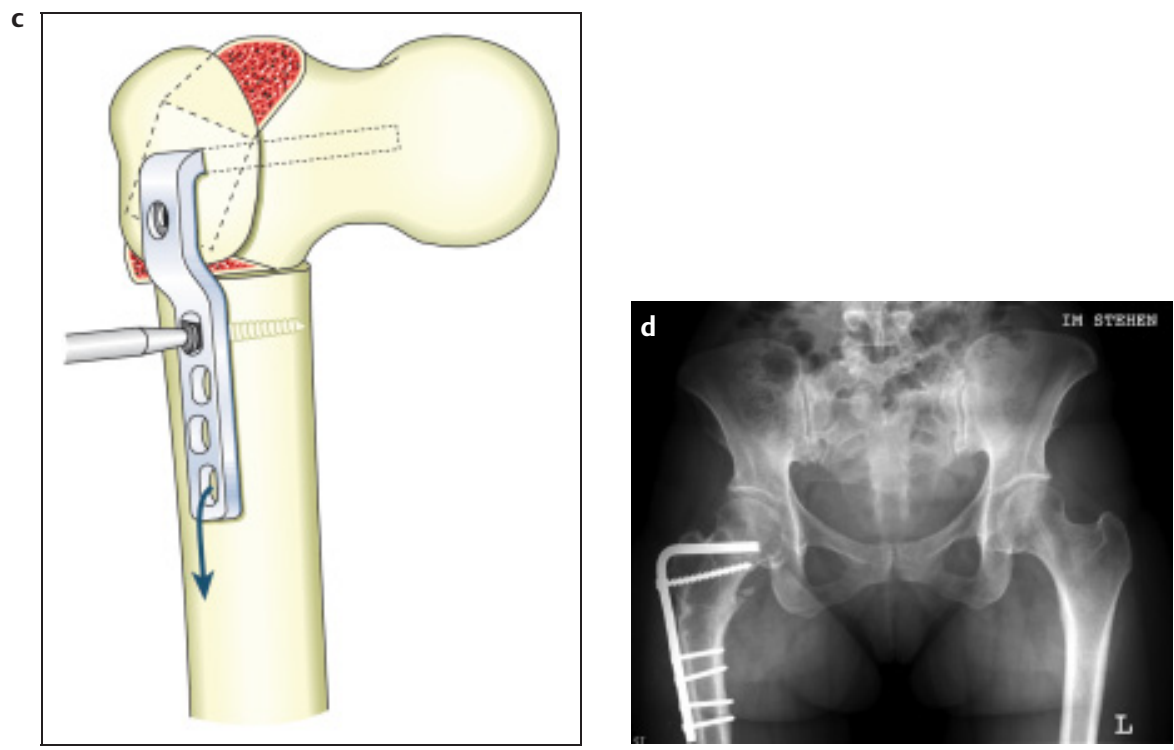

Abb.5c-d Zwei Jahre nach varisierender, trochanterversetzender (distalisierender), intertrochanterer Osteotomie: (c) Prinzip der Osteotomie nach Ganz. (d) Osteotomie problemlos verheilt. Weiterbestehendes hinkendes, aber wesentlich gebessertes Gangbild. Bis auf $30^{\circ}$ Innenrotationsdefizit bei $30^{\circ}$ Außenrotationsüberschuss freie Beweglichkeit. Bei Arbeitsfähigkeit besteht Wunsch nach Metallentfernung. tion. Heilung nach sofortiger Osteosynthese mit DHS. Kontinuierliche Schmerzsymptomatik mit Trendelenburg-Zeichen, Beugekontraktur von $15^{\circ}$ und Außenrotation des Oberschenkels. Ein Jahr nach der Osteosynthese extendierende, varisierende intertrochantere Osteotomie mit Trochanterversatz nach distal: sofortige Besserung der Beschwerden und Schmerzen, Besserung der Beweglichkeit, aber weiterbestehendes Trendelenburg-Zeichen. Im MRT Verfettung der pelvi-trochanteren Muskulatur als Zeichen der langfristigen Entlastung. Im CT nach Ausheilung noch $30^{\circ}$ Außenrotation des Oberschenkels nachweisbar. Die Patientin ist als Krankenschwester arbeitsfähig. Als nächster Eingriff ist eine innenrotierende Osteotomie zur Verbesserung des Muskelangriffspunktes für die pelvi-trochantere Muskulatur geplant (Abb.5a-h).

41-jähriger Patient mit seit Monaten zunehmenden Hüftschmerzen rechts. Chrogenaufnahmen Verdacht auf alkoholinduzierte Hüftkopfnekrose. Im MRT beginnende Hüftkopfnekrose rechts mit Minderperfusion des lateralen, belasteten Hüftkopfes. Gleichzeitig Nachweis einer schleichenden medialen Schenkelhalsfraktur. Daraufhin Stabilisierung der Schenkelhalsfraktur mit einer DHS und Implantation einer magnetfeldinduzierenden, zusätzlichen Schraube in die Nenischer Alkohomissbrauch. In den Rönt- 

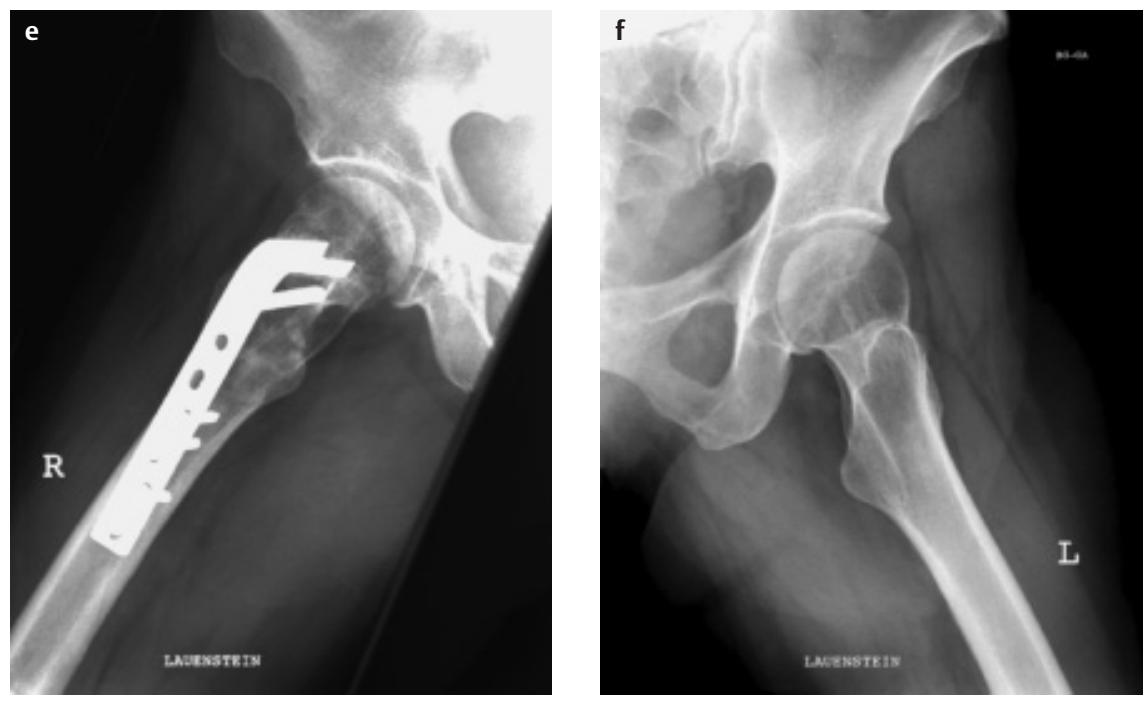

Abb.5 e-f Aufnahmen nach Lauenstein beider proximaler Oberschenkel: gute Kongruenz und Überdachung des Femurkopfes nach Varisierung rechts. Verkleinerung des Trochanter minor als Ausdruck einer verbliebenen Außenrotationsstellung.
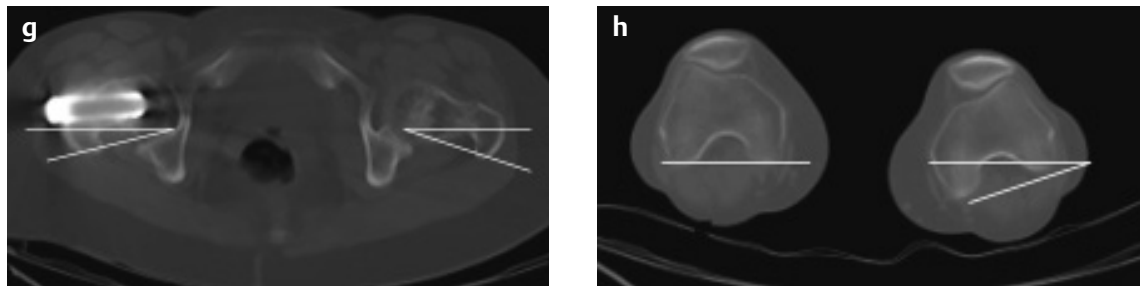

Abb.5 g-h CT-Aufnahmen zur Bestimmung der Rotation des Schenkelhalses: (g) Transversalaufnahmen der Schenkelhalsachse mit kompensatorischer Innendrehung rechts. (h) Transversalschnitt durch die Femurkondylen: verbliebene Außenrotation von $30^{\circ}$.
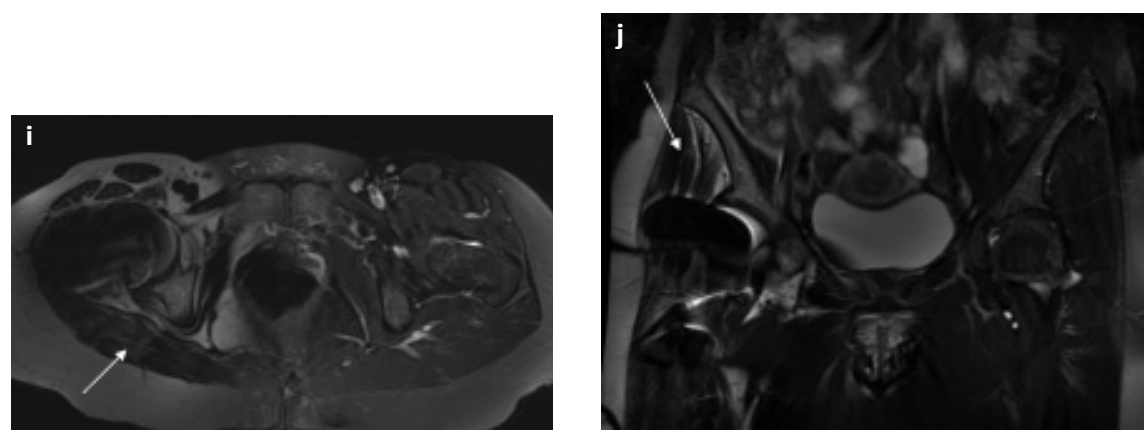

Abb.5 $\mathbf{i}-\mathbf{j} \quad$ MRT der pelvitrochanteren Muskulatur. (i) Transversalschnitt durch das Becken auf Höhe der Trochanteren: rechts Muskulatur vollständig atroph und ohne Spannung. Links Normalbefund. (j) Frontalschnitt mit Darstellung der verfetteten pelvitrochanteren Muskulatur, die durch die Außenrotation, Verkürzung und Valgisierung keine Spannung aufbauen kann. Artefakte durch Implantat. krosezone. Problemlose Ausheilung der Fraktur und Ausbleiben der Nekrose mit vollständiger Schmerzfreiheit nach 2 Jahren. Dann Beginn der Symptomatik auch in der linken Hüfte und Nachweis einer beginnenden Nekrose. Prophylaktisches Einbringen einer DHS und einer Schraube in die Zone der Minderperfusion (Abb.6a-g).

\section{Schlussfolgerung}

Die Schenkelhalsfraktur des jungen Erwachsenen geht einher mit einer hohen Komplikationsrate. Gelenk- und - wenn nicht möglich - wenigstens Knochenerhalt sind die Primäranforderungen bei diesen Patienten, da bei endoprothetischem Ersatz auch heute noch hohe Lockerungsraten beschrieben sind. Nach einer Wechseloperation ist ein weiterer Knochenverlust zu erwarten und die Verlustspirale dreht sich weiter. Die Möglichkeiten der konservierenden Chirurgie sind vielfältig und abgestuft einsetzbar.

Vor korrigierenden Operationen muss durch differenzierte Bildgebung eine Gesamtanalyse der vorliegenden Pathologie einschließlich der Weichteile erfolgen. Die Korrekturen können schrittweise oder in einer Sitzung angegangen werden. Die Auswirkungen von Korrekturen am Knochen auf die Weichteile (Muskelfunktion) müssen immer in Betracht gezogen werden.

Im Einzelnen sind folgende Verfahren, auch in Kombination sinnvoll:

- Anbohren des minderperfundierten Femurkopfareals (bone bruise)

- Einbringen einer Magnetfeldspule zur Induktion einer verbesserten Durchblutung über eine Schraube

- Perforation eines minderdurchbluteten Areals mit Bohrungen, Spongiosaunterfütterung, direkte Fixation der instabilen Zone.

- Osteochondrale autologe Transplantation (OATS)

- Umstellungsosteotomien aller Art

- Schenkelhalsverlängerung mit trikortikalem Span

- Knochensparende endoprothetische Behandlung 

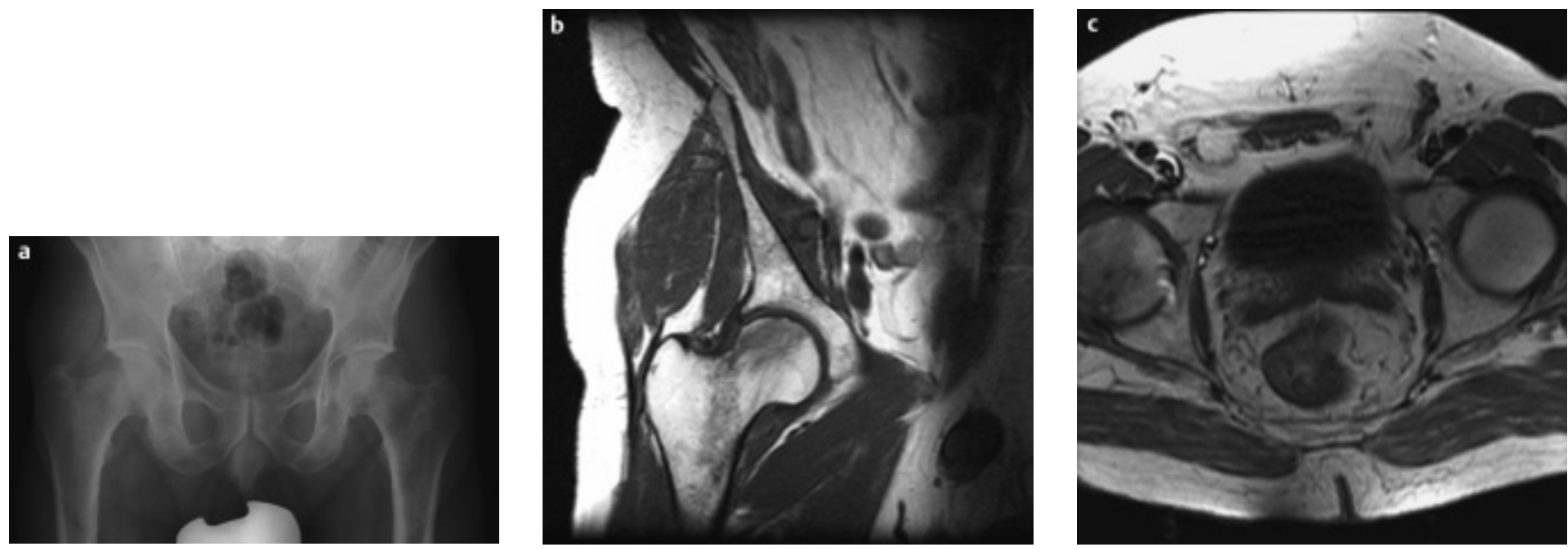

Abb.6 a-c (a) Beckenübersicht eines Patienten mit Alkoholanamnese ständige Hüftschmerzen rechts. Im MRT eindeutige Fraktur erkennbar. (b) Im frontalen MRT-Schnitt nicht dislozierte Schenkelhalsfraktur und

beginnende Femurkopfteilnekrose antero-lateral. (c) Im Vergleich der Sagittalschnitte durch beide Hüftköpfe erkennbare Signalalteration des rechten Hüftkopfes.
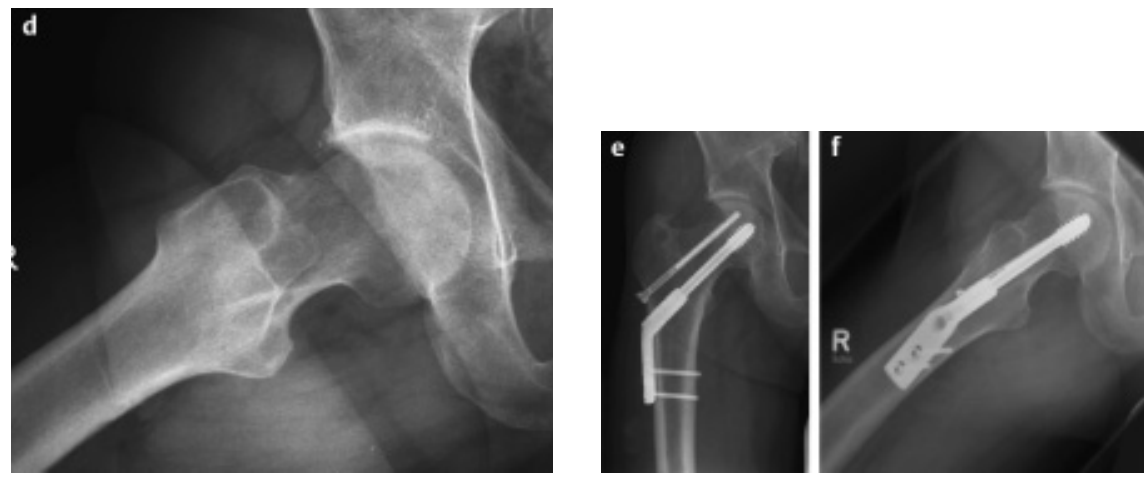

Abb.6 d-f (d) Lauenstein-Aufnahme rechtes proximalen Femur korrespondierend zur Teilnekrose lateral bei noch gut erhaltener Knochenstruktur ohne Hinweis auf Kollaps der Belastungszone. (e) Stabilisierung der Schenkelhalsfraktur mit einer DHS und Einbringen einer Zusatzschraube mit Induktion eines Magnetfeldes zur Verbesserung der lokalen Perfusion des nekrosegefährdeten Areals. (f) Lauenstein-Aufnahme zwei Jahre nach der Versorgung: Nekrose gestoppt. Keine Beschwerden, keine Bewegungseinschränkung.

\section{Literatur}

${ }^{1}$ Aldinger PR, Thomsen M, Mau H, Ewerbeck V, Breusch SJ. Cementless Spotorno tapered titanium stems: excellent $10-15$ survival in 141 young patients. Act Orthop Scand 2003; 74: $253-8$

${ }^{2}$ Bonnaire F, Kuner EH, Lorz W. Schenkelhalsfrakturen beim Erwachsenen: Gelenkerhaltende Operationen. Unfallchirurg 1995; 98 : 259-64

${ }^{3}$ Callaghan JJ, Forest EE, Sporer SM, Goetz DD, Johnston RC. Total hip replacement in the young adult. Clin Orthop 1997; 344: 257-62

${ }^{4}$ Crock V. An Atlas of vascular anatomy of the skeleton and spinal cord. Martin Dunitz 1956

${ }^{5}$ Daniel J, Pynsent PB, McMinn D J. Metal-onmetal resurfing of the hip in patients under the age of 40 years with osteoarthritis. J Bone Joint Surg Br 2004; 86: 177-84
${ }^{6}$ D’Antonio JA, Capello WN, Manley MT, Feinberg J. Hydroxylapatite coated implants. Total hip arthroplasty in the young patient and patients with avascular necrosis. Clin Orthop 1997; 344: $124-38$

${ }^{7}$ Garden RS. J Bone Joint Surg 1971; 53-B: $173-97$

${ }^{8}$ Huggler AH, Jakob AH, Bereiter H, Haferkorn M, Ryf C, Schenk R. Long-term results with the uncemented thrust plate prosthesis. Acta Orthop Belg 1993; 59: 215-23

${ }^{9}$ Iwase T, Hasegawa Y, Iwasada S, Kitamura S, Iwata $\mathrm{H}$. Total hip replacement after failed intertrochanteric valgus osteotomy for advanced osteoarthrosis. Clin Orthop 1999; 364: $175-81$

${ }^{10}$ Trueta J, Harrison MHM. The normal vascular anatomy of the femoral head in adult men. 1953 JBJS; 35 B: $442-48$

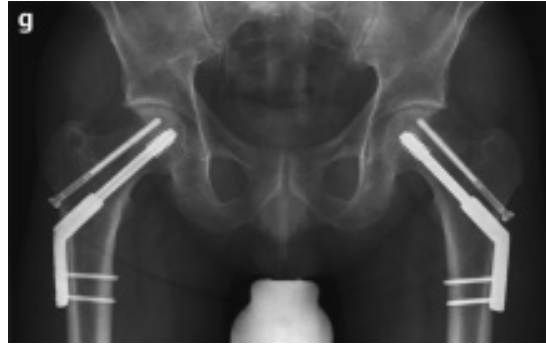

Abb.6g Patient klagt nach 2 Jahren über Hüftschmerzen links: Im MRT auch hier beginnende Femurkopfteilnekrose, allerdings ohne Schenkelhalsfraktur. Auch hier Indikation zur Stabilisierung des Schenkelhalses und Einbringen einer Magnetfeldspule.

Priv.-Doz. Dr. med. Felix Bonnaire Chefarzt

\section{Dr. med. Thomas Lein}

Oberarzt

Dr. med. Henning Röhl

AIP

Städtisches Klinikum Dresden-

Friedrichstadt

Klinik für Unfall-Wiederherstellungs-

und Handchirurgie

Friedrichstr. 41

D-01067 Dresden 